\title{
POLÍTICAS PÚBLICAS DE PARTICIPACIÓN CIUDADANA Y CAPITAL SOCIAL EN CHILE
}

Patrício Valdivieso

\begin{abstract}
Resumen
Alcanzadas ciertas condiciones mínimas para que la participación ciudadana sea posible, tales como son el imperio del orden político democrático y la superación de las desigualdades más extremas, es posible perfeccionar políticas e instrumentos que incrementen la calidad de la participación y promuevan el Capital Social. Mi principal hipótesis en este estudio es que los estados latinoamericanos disponen de instrumentos de participación ciudadana que podrían ser perfeccionados a fin de lograr una mayor integración y sinergia, especialmente en los espacios locales. A partir del análisis del caso de Chile, en este estudio ofrezco un diagnostico sobre los avances y obstáculos, y propuestas para la discusión y el progreso en las políticas de participación en el nivel local ${ }^{1}$.
\end{abstract}

Palabras clave: Participación Ciudadana; Local; Instrumentos; Chile.

\begin{abstract}
Assured some conditions for citizen participation, such as is the rule of democratic political order and overcoming extreme inequalities, it is possible to develop policies and tools that enhance the quality of participation. My main hypothesis in this study is that Latin American states have instruments of citizen participation that could be improved to achieve more integration and synergy, especially in local areas. From the analysis of the case of Chile, this study offers a diagnosis and proposals for discussion and progress in participation policies.
\end{abstract}

Keywords: Citizen Participation; Local; Tools; Chile.

\section{Introducción}

Durante las últimas tres décadas, la mayor parte de los gobiernos latinoamericanos ha centrado sus esfuerzos en promover iniciativas que fortalezcan la participación ciudadana y el Capital Social, generando condiciones destinadas a facilitar la participación democrática. Desde la introducción de reformas legales y administrativas hasta la incorporación de tecnologías a través de los portales web, hemos sido testigos del despliegue de una batería de instrumentos, cuya finalidad es incentivar a los individuos y a sus organizaciones para expresar sus opiniones y acceder a información pública que les permitan incidir en la toma de decisiones sobre asuntos de interés común. Asimismo, se han producido progresos en el ámbito comunal,

\footnotetext{
${ }^{1}$ El apoyo financiero para realizar este estudio es el proyecto FONDECYT Nr. 1110413.
} 
donde algunos gobiernos locales han generado prácticas estables de inclusión ciudadana. En algunos casos, el que hacer participativo ha traspasado el nivel discursivo y propositivo, posicionándose como rasgo distintivo en la forma de gestionar el gobierno local de ciertas autoridades municipales. Pero aún no han sido suficientes los esfuerzos para el despliegue de políticas integrales de participación ciudadana y resultados aceptables. El testimonio de los ciudadanos, recabado por numerosos estudios de opinión a lo largo y ancho de las Américas, sugiere que las políticas públicas y los instrumentos de participación no han arrojado aún resultados satisfactorios. Más aún, en el ámbito local de las comunas, las muestras de gestión participativa tienden a estar más vinculadas al sello distintivo que le impone la autoridad de turno a su gobierno que al imperativo intrínseco de la participación ciudadana como condición necesaria para el desarrollo local democrático y sustentable.

La principal hipótesis en este estudio es que las sociedades latinoamericanas disponen de instrumentos de participación que podrían ser perfeccionados a fin de lograr una mayor integración y sinergia, especialmente en los espacios locales. A partir del caso de Chile, en este estudio argumento que hay que mejorar los instrumentos públicos de participación ciudadana existentes y generar sinergias para que los esfuerzos ya desplegados muestren mejores resultados. Mi metodología consistió en recabar y sistematizar cuantiosa información dispersa sobre los instrumentos del Estado y la participación ciudadana en Chile, con el propósito de observar, detectar necesidades y formular propuestas para la discusión. Para observar empíricamente los niveles de vida asociativa, confianza y participación de los chilenos, hice uso de la información generada por medio de la aplicación de una encuesta sobre Capital Social en Santiago de Chile ${ }^{2}$. En este estudio parto por contextualizar las políticas chilenas de participación en las últimas décadas, sus progresos y debilidades. Posteriormente, observo, analizo y comento los grados de participación ciudadana, vida asociativa y confianza en el ámbito local, con el propósito de entender mejor el contexto social de la participación en la sociedad chilena y observar con precisión la brecha que existe entre las inquietudes y prácticas ciudadanas y las políticas púbicas. Finalmente propongo un conjunto de criterios, condiciones e iniciativas concretas con potencial de aportar en la discusión sobre la adecuación de los instrumentos públicos existentes para progresar en el horizonte de una participación local efectiva y habilitante.

\footnotetext{
${ }^{2}$ La encuesta fue aplicada en cinco comunas de la ciudad de Santiago el año 2005, a una muestra de 600 personas, para medir grados de participación y confianza. La información sobre políticas e instrumentos de participación fue recabada mediante diversos encuentros y focus group: tres talleres de trabajo en la Subsecretaría de Desarrollo Regional (SUBDERE) del Ministerio del Interior de Chile, cuatro reuniones con académicos, representantes de SUBDERE y de la Secretaría General de Gobierno, y numerosos otros encuentros más informales (reuniones, entrevistas). Asimismo, realicé actividades de observación con pautas en municipios de Santiago.
} 


\section{Políticas públicas y participación en Chile}

Al igual que en otros países de América Latina, desde la década de los noventa, los gobiernos chilenos han priorizado con distintos matices las metas del crecimiento, la internacionalización de la economía, el combate contra la pobreza y la consolidación y la profundización del sistema democrático, por medio de un ambicioso conjunto de reformas, que caben dentro de la categoría de "reformas de segunda generación", post consenso de Washington (1990). Particularmente importantes han sido las iniciativas para promover mayor descentralización, fortalecimiento de los gobiernos locales y participación ciudadana (SUBDERE, 2004; CARMONA, 2006). Los gobiernos han ido introduciendo distintas reformas legales y administrativas destinadas a incrementar el grado de autonomía local.

En el caso de Chile, en los discursos de los mensajes y de las cuentas públicas de las autoridades se puede observar un férreo compromiso con las políticas de participación ${ }^{3}$, y ha habido esfuerzos por promover una gestión más abierta y participativa de los servicios púbicos (VALDIVIESO et. al. 2003; SUBDERE, 2004; CARMONA, 2006). Variados programas, incentivos legales, económicos y sociales han sido diseñados e implementados con el propósito de promover la participación en los espacios locales. Así se han desarrollado numerosas iniciativas, donde interactúan ministerios, gobiernos locales (municipios), programas sociales, universidades y la comunidad local; por ejemplo, fondos concursables para el financiamiento de proyectos sociales de iniciativa comunal, programas participativos en vivienda y urbanismo, salud, educación e infraestructuras.

Desde la década de 1980, las políticas de regionalización y desconcentración de la administración pública han otorgando un papel central a los municipios por medio de la transferencia de atribuciones y servicios en los ámbitos de educación (escuelas públicas de enseñanza primaria y secundaria), salud (atención primaria), seguridad e infraestructuras. La estrategia de la década de 1990 no sólo reforzó y profundizó la desconcentración, sino que aumentó las competencias y responsabilidades municipales en diversos ámbitos del desarrollo social y político. En la década del 2000, la declaración oficial del gobierno enfatizó el importante papel que cabía a la sociedad civil en los espacios locales, en numerosos temas, desde la superación de la pobreza hasta la seguridad ciudadana, y ello se tradujo en nuevas iniciativas encaminadas a fortalecer los municipios. Sumado a este esfuerzo estatal, desde las universidades y las organizaciones de la sociedad civil surgieron interesantes ejemplos de apoyo a esta labor. Por medio de la metodología de concursos, se distinguió y reconoció iniciativas innovadoras y buenas prácticas dignas de ser replicadas.

\footnotetext{
${ }^{3}$ Discursos disponibles en: <www.presidencia.cl>. Acceso en: 31 may. 2011.
} 
A principios de la década del 2000, el presidente Ricardo Lagos (20002006) confirmó la voluntad de su Gobierno de fortalecer la participación ciudadana en la gestión pública, comprometiendo cinco líneas de acción gubernamental para hacerla efectiva: (i) generar iniciativas hacia una gestión pública participativa; (ii) incorporar la participación ciudadana en el diseño y evaluación de las políticas y programas públicos; (iii) promover acciones para el fortalecimiento de las organizaciones de la sociedad civil; (iv) promover la articulación permanente entre sociedad civil y Estado; ( $v$ ) promover los vínculos regionales y locales para la promoción de los derechos ciudadanos (Proyecto "Ley Bases de Participación Ciudadana en la Gestión Pública"; Ministerio Secretaria General de Gobierno, 2002 ${ }^{4}$ ). Su sucesora, Michelle Bachelet (2006-2010) priorizó también el tema de la participación en relación con las políticas sociales del gobierno, sumando nuevos programas e instrumentos tales como las cuentas públicas participativas, diversas iniciativas de democracia directa para la asignación de recursos municipales (presupuestos participativos, cartas ciudadanas, etc.), el fondo de desarrollo de la sociedad civil, la agenda pro-ciudadana, y los portales web ciudadanos ${ }^{5}$. El actual Presidente Sebastian Piñera (2010-2014) se ha comprometido a practicar el principio de la subsidiariedad en las dimensiones regionales y municipales, y a favorecer la participación ciudadana y de la sociedad civil en las políticas orientadas a incrementar las oportunidades, las seguridades y fortalecer los valores que debiesen dar mayor cohesión y proyección al país ${ }^{6}$.

A pesar de los esfuerzos y las acciones desplegadas, hay razones justificadas para preguntar si ha sido suficiente. La insatisfacción ciudadana y las protestas sociales demuestran que sigue existiendo una gran brecha, difícil de acortar, entre la visión deseada de "gobierno cercano a la gente", eslogan frecuente las autoridades contemporáneas, y los procesos y las prácticas de participación en relación con las políticas, los programas, las medidas y las acciones, y por sobre todo sus resultados. Por lo menos existen cuatro factores que afectan las iniciativas gubernamentales e impiden que las políticas resulten coronadas por niveles de participación ciudadana aceptables, entendiendo por ella la vida asociativa, la confianza y la participación de las personas, sus organizaciones y sus comunidades en los espacios públicos, institucionales y programas.

\footnotetext{
${ }^{4}$ Disponible en: <http://www.innovacionciudadana.cl/portal/imagen/File/barometro/Informe\%20 final\%20S.E.pdf>. Acceso en: 31 may. 2011.

5 Disponible en: <http://www.participemos.cl>. Acceso en: 29 jun. 2011. Disponible en: <http://www.portalciudadano.cl>. Acceso en: 29 jun. 2011.

6 Véase Programa de Gobierno y Discurso del 21 de mayo del 2010. Disponible en: $<$ http://www.gobiernodechile.cl/programa-de-gobierno/>. Acceso en: 29 jun. 2011. Disponible en: <http://www.gobiernodechile.cl /especiales/mensaje-presidencial-21-de-mayo/>. Acceso en: 29 jun. 2011.
} 
Falta de recursos presupuestarios y humanos, tanto en cantidad como en cuanto a su preparación. En los servicios públicos se observa asimetrías en recursos y condiciones para lograr buenos resultados. No obstante las capacidades de algunos servicios, otros no cuentan con los recursos humanos y materiales necesarios para hacer lo propio; la SUBDERE del Ministerio del Interior dispone de recursos humanos y económicos para realizar estudios en el nivel nacional, central, mientras que la División de Organizaciones Sociales de la Secretaría General de Gobierno, encargada de hacer seguimiento de más de ciento cincuenta servicios de participación en todo el país, sólo tiene un pequeño grupo de funcionarios para ordenar información que llega incompleta de los mismos servicios y con retraso, y la mayor parte de los municipios no dispone de recursos ni de personal preparado.

Carencia de coordinación y colaboración entre instituciones. Desde los niveles centrales hasta las esferas locales interactúan numerosas instancias y organizaciones, sin existir condiciones que garanticen sinergias en las cadenas de trabajo, ni tampoco sistemas de seguimiento que faciliten el cumplimiento de las funciones para que el todo se retroalimente y progrese. Bajo tales condiciones, descoordinación y falta de cooperación, que se suman a la falta de recursos e incentivos, no es posible esperar que las entidades públicas involucradas estén en condiciones de cumplir bien actividades y rutinas que son esenciales para que los programas de participación ciudadana y comunitaria realmente tengan buenos resultados.

Ausencia de una cultura organizacional apropiada que promueva la participación. El volumen de tareas ha ido aumentando en el ámbito regional y comunal, a partir de las transferencias de competencias desde el nivel central a las regiones y a los municipios. Bajo condición de falta de recursos y disfuncionalidad intersectorial, ello genera desgaste en los funcionarios y en la cultura organizacional. El problema de la cultura organizacional, sumado a los cambios recurrentes que se producen en los niveles políticos de los procesos de toma de decisión, hace imposible el adecuado seguimiento y perfeccionamiento. Así, numerosos programas pierden actualidad, quedan reducidos a ser un legado de ciertas autoridades políticas que circulan en la administración pública. Otros desaparecen, cuando cambian radicalmente las prioridades de quienes asumen cargos de responsabilidad y toma de decisiones.

Débil autonomía y autogestión en los municipios. En la mayor parte de los municipios la falta de autonomía de los programas y servicios que tienen un fuerte componente participativo debilita el impacto esperado. Los estilos de las autoridades de turno y los cambios de los altos funcionarios determinan prioridades, énfasis y decisiones centrales en relación con esos programas, afectando muchas veces la continuidad de las políticas de participación. 
Además, las relaciones asimétricas de poder que se establecen en un Estado presidencialista y unitario como es el chileno, promueven comportamientos de alta dependencia, asistencialismo, clientelismo y relaciones verticales que juegan en contra de lógicas de autogestión y autonomía local.

En el día a día, la falta de recursos, capital humano, coordinación, la cultura y las prácticas heredadas, la gran cantidad de labores a realizar y la falta de autonomía local conspiran contra numerosas iniciativas y el buen funcionamiento de los servicios y programas, provocando que el impacto de los esfuerzos realizados desde el Estado se vean disminuidos, y que ello encuentre su correlato, según veremos más adelante, en bajos niveles de vida asociativa, confianza y participación ciudadana en las comunas.

\section{Los municipios}

En Chile existe un reconocimiento generalizado sobre la importancia de los municipios en los procesos de desarrollo en el nivel local (BAEZA, 2004; CARMONA, 2006; VALDIVIESO, 2008). Dentro de sus atribuciones está la administración de servicios esenciales para la calidad de vida de la población. Por lo tanto, el buen funcionamiento, la transparencia y la participación en el gobierno local son factores que influyen en las condiciones de vida de las personas, y al mediano plazo en la gobernabilidad. De ahí el consenso en torno a la necesidad de fortalecer la participación en los espacios locales y hacer más eficientes los servicios.

Las municipalidades disponen de instrumentos y mecanismos para el fortalecimiento de la participación. La Constitución de Chile y otras normas contienen numerosas disposiciones que regulan prácticas de participación. En las regiones, los Consejos Regionales, integrados por consejeros elegidos por concejales de las municipalidades, disponen de instrumentos legales para intervenir en el desarrollo local vía decisiones sobre la inversión regional, y pueden hacer efectiva la participación de la comunidad (art. 28 de Ley 19.175 sobre administración del Estado). En las provincias, los Consejos Económicos Sociales Provinciales, integrados por representantes de organizaciones sociales, pueden representar la participación organizada. Además, los municipios cuentan con diversos procedimientos e instancias para canalizar la participación. Los alcaldes y los concejales son de elección directa, y los primeros elaboran cuentas anuales, mantienen audiencias públicas, realizan consultas, pueden practicar plebiscitos e incluir mecanismos de participación en los procesos de decisión presupuestaria. Por otra parte, dentro del municipio hay oficinas e instancias que pueden canalizar las inquietudes ciudadanas. Las ordenanzas de participación (art. 93 de ley 18.695 de municipalidades) establecen modalidades y formas concretas de interacción de los municipios con los vecinos, según el contexto particular de cada municipalidad. Asimismo, los municipios, en conjunto otras instancias 
institucionales, participan en programas sectoriales de desarrollo local, y disponen de fondos para conectar las políticas de desarrollo local con la participación. Las juntas de vecinos y las organizaciones comunitarias (reguladas por la Ley 19.418) tienen por función representar inquietudes ciudadanas (comunitarias) en los municipios. Ellas debieran representar, informar, comunicar inquietudes e iniciativas, colaborar en programas y servicios (medio ambiente, salud, educación, arte, cultura, deporte, urbanismo y hábitat, seguridad, combate a la pobreza, etc.).

No obstante lo anterior, en línea con los problemas que afectan al Estado, en el mundo municipal chileno hay un conjunto de obstáculos que dificultan la marcha de servicios y programas municipales representativos y eficaces, todo ello en perjuicio de la participación. Entre estos, cabe mencionar:

- $\quad$ Carencia de orientaciones claras. En el país y en los municipios coexisten distintas visiones y comprensiones sobre la participación ciudadana, lo cual se traduce en diversidad y dispersión de criterios para tomar y ejecutar decisiones. En dos municipios distintos, la participación puede llegar a ser entendida en sentido opuesto; para un Alcalde puede consistir en reunir gente para que escuche su cuenta o discurso, mientras que para otro puede ser convocar a los dirigentes de las juntas de vecinos para que diseñen planes de desarrollo comunal. La diversidad y la dispersión de criterios explica una enorme variedad de iniciativas programáticas e intervenciones que se describen como "participativas", sin llegar a ser claro en qué sentido lo son, qué instrumentos de medición incorporan, y si éstos son adecuados para observar grados de intervención de los ciudadanos y de sus organizaciones en las políticas y programas locales.

- $\quad$ Carencia de espacios para acoger inquietudes y preferencias por la participación. No obstante existir numerosos programas y mecanismos prediseñados desde el nivel central de gobierno, faltan instrumentos y espacios municipales que entren en sintonía con las preferencias y posibilidades reales de participación de los ciudadanos concretos que allí habitan, según sus contextos socioeconómicos, culturales y características territoriales específicas. Ante la falta de un espacio real de participación autónoma, visible para las personas en el día a día, que facilite el empoderamiento y la generación de capacidades para intervenir, es difícil que aumente el interés y la confianza en las instituciones y programas públicos.

\footnotetext{
Carencia de instrumentos y metodologías para identificar demandas de manera integral y especializada territorialmente. Los instrumentos usados por los servicios públicos centrales y municipales que generan información sectorial sobre las demandas ciudadanas dan lugar al diseño de diagnósticos desde perspectivas temáticas predominantes en los
} 
propios servicios o en la esfera de las autoridades y de los actores locales dominantes. Esos diagnósticos sesgados consideran sólo una parte de la realidad, quedando fuera causales, relaciones y factores que pueden ser de primera importancia para los resultados, tales como son las particularidades que distinguen a los territorios y las comunidades que ahí habitan. En todo orden de cosas tiende a predominar una lógica sectorial que empobrece la perspectiva para las intervenciones. Por consiguiente, en la realidad local se van superponiendo intervenciones parciales, de fácil y rápida implementación, cuyos efectos colaterales no son previsibles. Tal aproximación y los procedimientos relacionados determinan la priorización en el uso de los recursos y los efectos de corto plazo. Al no representar soluciones integradas en las realidades locales, los efectos de los programas y de las intervenciones son muchas veces desafortunados o contradictorios. La falta de una política que integre visiones y perspectivas se refleja en la descoordinación, la duplicidad de funciones, el traslape de programas de intervenciones, la coexistencia de una variedad de instrumentos que duplican miniformación. Todo lo anterior significa un elevado gasto de recursos y da lugar a una sobre intervención en las comunas y localidades más pequeñas. Cada institución realiza su propio diagnóstico y cada diagnóstico justifica la creación de un nuevo programa o instrumento de intervención. Sin embargo, la población en la que se aplican las encuestas, entrevistas, talleres que permiten recabar información para el diseño de programas o instrumentos sectoriales sigue siendo la misma. Con el paso del tiempo, las personas van perdiendo la confianza en la institucionalidad pública, confundiendo los mensajes y propósitos ante la variedad de visitas, informaciones y autoridades que enfatizan distintas cosas, que finalmente no entran en sintonía con sus intereses y necesidades.

- Diferencias en los tiempos y en los ritmos. En los municipios chilenos se manifiesta un problema que atraviesa toda la administración del Estado, a saber, resulta extremadamente difícil, sino imposible, coordinar los tiempos y ritmos de los gobiernos y las administraciones locales y regionales con los tiempos requeridos para el desarrollo de procesos participativos que sean genuinamente inclusivos. Por una parte, la presión constante de los gobiernos por cumplir las promesas de los discursos, los compromisos coyunturales, la cercanía de las elecciones o períodos asociados a la captación de votos por medio de obras tangibles, entre otros, dificultan el gradual curso que debiese tomar una intervención participativa o un proyecto de desarrollo comunal. Por otra parte, el bajo grado de vida asociativa hace necesaria la inversión de recursos y de tiempo en tareas previas a cualquier intervención, tales como son identificar a los actores estratégicos, generar confianza, validar los instrumentos para legitimar acciones futuras, organizar a los actores y concertar acuerdos de trabajo colectivo, crear y estimular canales o mecanismos apropiados para promover el diálogo y debate entre los actores, 
y fortalecer diversas competencias necesarias para un trabajo gradual y de largo aliento.

\section{Participación ciudadana y vida asociativa}

Dadas las condiciones políticas e institucionales descritas, no es extraño que en Chile se observe bajo nivel de vida asociativa, que la confianza en las instituciones sea baja, al igual que el interés por la política. A continuación describiremos los resultados obtenidos por medio de la aplicación de una encuesta en cinco comunas de Santiago de Chile el año $2005^{7}$, en relación con lo anterior.

El año 2005, el 73\% de las personas tenía poco o ningún interés por la política y por los asuntos públicos. Mediciones posteriores reiteraron esa tendencia ${ }^{8}$; por ejemplo, el año 2007 , sólo el $23,8 \%$ se informaba regularmente sobre asuntos públicos.

Cuadro 1 - Participación Política y vida asociativa, en $(1995,2004,2005,2006,2007,2009)(\%)$

\begin{tabular}{|l|c|c|c|c|c|c|c|c|}
\hline & $\mathbf{1 9 9 5}$ & $\mathbf{2 0 0 0}$ & $\mathbf{2 0 0 3}$ & $\mathbf{2 0 0 4}$ & $\mathbf{2 0 0 5}$ & $\mathbf{2 0 0 6}$ & $\mathbf{2 0 0 7}$ & $\mathbf{2 0 0 9}$ \\
\hline Votar en elecciones & & & & & 72,0 & 76,0 & 74,0 & 70,0 \\
\hline $\begin{array}{l}\text { Participación en } \\
\text { voluntariado }\end{array}$ & & 1,3 & 2,1 & & 10,4 & 8,9 & 2,0 & 2,3 \\
\hline $\begin{array}{l}\text { Participación en grupos } \\
\text { de interés y } \\
\text { organizaciones } \\
\text { utilitarias }\end{array}$ & 19,1 & 18,7 & & 12,0 & 13,7 & 3,0 & 13,2 \\
\hline $\begin{array}{l}\text { Participación en } \\
\text { asociaciones religiosas }\end{array}$ & & 6.6 & 8,0 & & 26,2 & 25,5 & 13,2 & 11,4 \\
\hline $\begin{array}{l}\text { Participación en } \\
\text { acciones colectivas } \\
\text { desafiantes }\end{array}$ & & & & & 17,2 & 15,8 & 15,7 & 19,4 \\
\hline Partidos & 2,5 & & & 2,0 & 2,8 & 2,4 & 0,1 & 4 \\
\hline Sindicatos & 3,4 & & & 5,1 & 1,8 & 3,4 & 2,6 & 2,6 \\
\hline Juntas de vecinos & 3,6 & & & 3,37 & 1,8 & 11,1 & 4,4 & 4,6 \\
\hline
\end{tabular}

Fuente: cuestionarios, índices y bases de datos de la pesquisa: Social Capital and Local Participation in South America: Data, Facts and Analysis ${ }^{9}$.

En el Cuadro 1, la vida asociativa tiende a ser baja, en todos los años y en todas las categorías, destacando la participación en acciones colectivas desafiantes (por ejemplo protestas) y en asociaciones religiosas.

\footnotetext{
${ }^{7}$ Encuesta y bases de datos disponibles en <http://www.capital-social.com/wp/?page_id=251\& preview=true $>$. Acceso en: 22 jun. 2011.

${ }^{8}$ Disponibles en: <http://www.capital-social.com>. Acceso en: 22 jun. 2011.

${ }^{9}$ Disponibles en: <http://www.capital-social.com/wp/?\%20page_id=251\&preview=true>. Acceso en: 22 jun. 2011.
} 
Comparativamente, los porcentajes de vida asociativa en otras formas de organización social, tales como organizaciones comunitarias, juntas de vecinos, partidos políticos y sindicatos tiende a ser bajo. El hecho que esas organizaciones tengan pocas personas inscritas y que participen tiene relación con sus dificultades para articular intereses ciudadanos y representar.

En las medición que realicé en Chile $(2005)^{10}$, buena parte de quienes tienen baja o nula vida asociativa responden que se debe a la falta de tiempo, falta de interés, motivos personales, falta de credibilidad, falta de percepción de los beneficios que puede traer la participación. El desinterés no es necesariamente ausencia de inquietudes o la apatía por hacer cosas junto a otros, esa actitud puede estar referida a la indiferencia y a la falta de motivación por ofertas que no aportan mucho a las necesidades cotidianas de las personas y de sus seres cercanos. En este sentido, probablemente ello tenga relación con la falta de percepción en los beneficios.

La informaciones del Cuadro 2, sobre las razones de la participación, sugieren que los bajos niveles de asociativismo podrían tener relación con falta de sintonía entre las ofertas de participación y las inquietudes de de las personas, en una sociedad crecientemente pluralista en intereses, convicciones y estilos de vida, compuesta por personas que día a día manifiestan un mayor grado de individualismo u utilitarismo en sus preferencias y decisiones. Para ilustrarlo de forma muy concreta, con un par de preguntas retóricas: ¿qué eco podrá encontrar en un joven la oferta de participación ciudadana en cuestiones municipales o en una junta de vecinos, si su inquietud del día a día consiste en trabajar para ganar más dinero con el propósito de poder subsistir o tratar de acceder a mejores condiciones de vida por medio del financiamiento de sus estudios o maximizar su tiempo libre? ¿hasta qué punto es esperable que una asociación comunitaria de barrio centrada en temas ciudadanos de interés común cautive el interés de una madre que tiene como primera prioridad obtener ingresos y no perder su precario empleo, quien apenas tiene tiempo para destinar a su familia en su hogar?

${ }^{10}$ Disponibles en: <http://www.capital-social.com/wp/?page_id=251\&preview=true>. Acceso en: 22 jun. 2011. 
Cuadro 2 - Razones declaradas de la participación (2005)

\begin{tabular}{|l|c|}
\hline \multicolumn{1}{|c|}{ RAZONES } & $\mathbf{\%}$ \\
\hline Espiritual, posición social, autoestima & 12,0 \\
\hline Placer o diversión & 9,6 \\
\hline Trae beneficios a la comunidad & 5,0 \\
\hline Otro & 1,8 \\
\hline Mejorar el sueldo familiar o acceso a servicios & 1,6 \\
\hline Es importante en situaciones de emergencia en el futuro & 0,4 \\
\hline No responden (son los que no participan de grupos) & 69,6 \\
\hline ToTAL & 100,0 \\
\hline
\end{tabular}

Fuente: cuestionarios, índices y bases de datos de la pesquisa: Social Capital and Local Participation in South America: Data, Facts and Analysis ${ }^{11}$.

La declaración de "falta de credibilidad" como motivo de no participar en las instancias de la vida asociativa, probablemente encuentre su explicación en malas experiencias de participación ${ }^{12}$. Este fenómeno suele ser el resultado de dos tipos de quiebres, un quiebre horizontal que se produce por la acción de los dirigentes que hacen uso de las asociaciones para perpetuarse en los cargos de dirección y obtener beneficios individuales, sin promover la rotación y la renovación. Un quiebre vertical de tipo generacional, por el cual los jóvenes sin contar con espacios reales para ser interlocutores y participar tienden a no participar. Formalmente puede haber numerosas organizaciones con estatutos al día, dirigentes que son visibles e instrumentos de difusión, pero las bases sociales de esas organizaciones por lo general son débiles o inexistentes.

\section{Confianza y cultura política}

En la literatura especializada hay un amplio consenso sobre la centralidad de la confianza y de las actitudes cívicas habilitantes para la vida asociativa y la participación ciudadana en la democracia (ver COLEMAN, 1990; PUTNAM, 1993; NORRIS, 2002; BAQUERO, 2005).

El Cuadro 3 muestra que en Chile la familia y la Iglesia Católica continúan disfrutando de un alto grado de confianza. El 2005, el $84,4 \%$ de los encuestados declaraba que en una situación de emergencia, por ejemplo en caso de enfermedad de una persona cercana o frente a la pérdida del empleo, recibiría ayuda de sus familiares.

\footnotetext{
11 Disponibles en: <http://www.capital-social.com/wp/?\%20page_id=251\&preview=true>. Acceso en: 22 jun. 2011.

12 En el marco de ésta y de otros estudios comparados con Brasil (VALDIVIESO, 2009), en diversos encuentros con dirigentes comunales y de distintas organizaciones, y con jóvenes de diversas edades, he podido detectar un fuerte divorcio entre los dirigentes y los asociados (véase numerosos documentos de trabajo en sección "resultados parciales", disponibles en: <http://www.puc.cl/icp/eticapolitica/>, acceso en: 22 jun. 2011).
} 
A SITUAÇÃO DAS AMÉRICAS:

DEMOCRACIA, CAPITAL SOCIAL E EMPODERAMENTO

Cuadro 3 - Confianza en instituiciones: Mucho, Poco, Nada (2005, 2008, 2009) (\%)

\begin{tabular}{|l|c|c|c|}
\hline & Mucho & Poco & Nada \\
\hline Congreso & 4,2 & 54,2 & 40,0 \\
\hline Gobierno & 16,4 & 54,4 & 28,8 \\
\hline Juntas vecinos & 11,8 & 39,4 & 43,8 \\
\hline Municipalidad & 12,2 & 51,4 & 35,0 \\
\hline Justicia & 9,0 & 45,4 & 44,2 \\
\hline Partidos & 2,6 & 34,6 & 61,6 \\
\hline Policia & 19,4 & 56,8 & 23,6 \\
\hline Iglesia & 50,2 & 30,8 & 18,2 \\
\hline Familia & 89,8 & 8,2 & 1,8 \\
\hline Vecinos & 28,8 & 48,2 & 21,4 \\
\hline Asociaciones comunitarias & 12,4 & 49,8 & 31,8 \\
\hline Sindicatos & 9,4 & 35,8 & 45,2 \\
\hline Medios & 21,2 & 51,4 & 26,6 \\
\hline
\end{tabular}

Fuente: Encuestas de elaboración propia y bases de datos de la pesquisa: Social Capital and Local Participation in South America: Data, Facts and Análisis ${ }^{13}$.

La Iglesia Católica tiene gran influencia en la sociedad, principalmente por el cumplimiento de numerosas funciones centrales en el ciclo vital de sectores significativos de la población, sus actividades de educación y de beneficencia pública. Cabe consignar también que un gran número de fiestas religiosas son nacionales y que la Iglesia Católica tiene representación garantizada en los eventos públicos de mayor repercusión en el país. Todas esas razones explican, en parte, la gran confianza de las personas en la Iglesia Católica. Por otra parte, según se ha observado por el lado de la vida asociativa, otras grandes organizaciones institucionalizadas, antaño con gran prestigio y arraigo en la población chilena, pasan por una situación difícil en la actualidad; tal es el caso de los partidos políticos y los sindicatos. A su turno, las principales instituciones del país, el gobierno, el poder legislativo y el poder judicial, las municipalidades, tienen discretos niveles de aprobación y de confianza.

Un significativo porcentaje de los encuestados, Cuadro 4, manifestaba bajos niveles de confianza interpersonal. Cuando las preguntas estaban referidas a la confianza mutua entre individuos, entre unos y otros, la mayoría manifestaba una actitud "hobbesiana". En cuanto a ayuda mutua o cooperación, sólo el $55,6 \%$ de los encuestados consideraba que podía contar con los vecinos para ocuparse de su hogar y de sus hijos en caso que debiese viajar por unos días, y sólo el $12,6 \%$ consideraba que recibiría ayuda. Por otra parte, sólo el $38,2 \%$ pensaba que se puede esperar ayuda en el barrio. En el caso hipotético de una situación muy difícil, por ejemplo enfermedad de una

${ }^{13}$ Disponibles en: <http://www.capital-social.com/wp/?page_id=251\&preview=true>. Acceso en: 22 jun. 2011. 
persona cercana o pérdida del empleo, sólo el $43,8 \%$ esperaba recibir apoyo de sus vecinos, y el $31,2 \%$ de sus compañeros de trabajo. Estos resultados no debieran sorprender, puesto que en otros estudios realizados en Chile y en otros lugares del mundo los niveles de confianza tampoco son altos (ver NORRIS, 2002; VALDIVIESO, 2009).

Cuadro 4 - Confianza en el nivel local y de las relaciones interpersonales in Chile (2005)

\begin{tabular}{|l|c|c|c|c|}
\hline \multicolumn{1}{|c|}{ Afirmaciones } & \multicolumn{3}{|c|}{ Alternativas de respuesta } \\
\cline { 2 - 5 } & $\mathrm{Si}$ & $\begin{array}{c}\text { Algunas } \\
\text { veces }\end{array}$ & Nunca & $\mathrm{Ns-Nr}$ \\
\hline $\begin{array}{l}\text { La mayoría de las personas del } \\
\text { barrio generalmente se } \\
\text { encuentra dispuesta a ayudarlo } \\
\text { en caso de necesidad }\end{array}$ & 38,2 & 32,8 & 23,4 & 5,6 \\
\hline $\begin{array}{l}\text { Se puede confiar en la mayoría } \\
\text { de las personas que viven en } \\
\text { su barrio }\end{array}$ & 24,2 & 42,4 & 31,8 & 1,6 \\
\hline $\begin{array}{l}\text { En este barrio es necesario } \\
\text { estar atento porque alguien } \\
\text { puede sacar ventaja de } \\
\text { Usted }\end{array}$ & 58,2 & 17,8 & 20,8 & 3,2 \\
\hline $\begin{array}{l}\text { Las personas de barrio en } \\
\text { general no confían unas en las } \\
\text { otras para prestar Dinero }\end{array}$ & 51,2 & 10,2 & 26,2 & 12,4 \\
\hline
\end{tabular}

Fuente: Encuestas de elaboración propia y bases de datos de la pesquisa: Social Capital and Local Participation in South America: Data, Facts and Análisis ${ }^{14}$.

\section{Relaciones entre vida asociativa, confianza $y$ otras variables relacionadas}

Las informaciones generadas por medio de la encuesta del año 2005 arrojaron algunas correlaciones de interés. La participación política, la vida asociativa y la confianza estaban correlacionadas entre sí.

\footnotetext{
${ }^{14}$ Disponibles en: <http://www.capital-social.com/wp/?page_id=251\&preview=true>. Acceso en: 22 jun. 2011.
} 
Cuadro 5 - Participación Política, variables socio-económicas y de Cultura Política (correlaciones, 2005)

\begin{tabular}{|c|c|c|c|}
\hline & $\begin{array}{l}\text { PARTICIPACIÓN } \\
\text { POLÍTICA (IPP) }\end{array}$ & $\begin{array}{c}\text { DISCUTE SOBRE } \\
\text { POLÍTICA (IDSP) }\end{array}$ & $\begin{array}{l}\text { INFORMARSE } \\
\text { (IISP) }\end{array}$ \\
\hline Sexo & $-.08^{*}$ & $-.11^{*}$ & $-.08 *$ \\
\hline Nivel estudios & $.09 *$ & $.26 * * *$ & n.s. \\
\hline Nivel ingreso & $.14^{* *}$ & $.19 * * *$ & n.s. \\
\hline Bienestar personal & $.13 * *$ & n.s. & n.s. \\
\hline Expectativas de vida & $.11^{*}$ & $.10 *$ & n.s. \\
\hline Calidad de vida & $.19 * * *$ & $.17 * * *$ & n.s. \\
\hline $\begin{array}{l}\text { Infraestructuras } \\
\text { asociativas }\end{array}$ & $.15^{* *}$ & $.16 * * *$ & $.08^{*}$ \\
\hline Inseguridad & $-.15 * * *$ & n.s. & $-.08 *$ \\
\hline $\begin{array}{l}\text { Satisfacción con la } \\
\text { democracia }\end{array}$ & $.21 * * *$ & n.s. & n.s. \\
\hline $\begin{array}{l}\text { Solidaridad e } \\
\text { identificación }\end{array}$ & $.21 * * *$ & $.24 * * *$ & $.14^{* *}$ \\
\hline Sentido colectivo & n.s. & n.s. & n.s. \\
\hline Apoyo a la democracia & n.s. & $.20 * * *$ & n.s. \\
\hline Participación política & --- & $.37 * * *$ & $.31 * * *$ \\
\hline Discutir sobre política & $.38 * * *$ & --- & $.47 * * *$ \\
\hline Informarse & $.31 * * *$ & $.47 * * *$ & --- \\
\hline
\end{tabular}

Nivel de significación: n.s. indica $>0,1 ; *$ indica $\mathrm{p}<0,1 ; * *$ indica $\mathrm{p}<0,05 ; * * *$ indica $\mathrm{p}<0,01$ Fuente: Encuestas de elaboración propia y bases de datos de la pesquisa: Social Capital and Local Participation in South America: Data, Facts and Análisis ${ }^{15}$.

El Cuadro 6 muestra correlaciones entre diversas formas de participación y otras variables demográficas y socioeconómicas.

Cuadro 6 - Participación en asociaciones, acciones colectivas desafiantes, variables socio-econômicas y cultura política (correlaciones, 2005)

\begin{tabular}{|c|c|c|c|c|}
\hline & $\begin{array}{l}\text { PARTICIPA } \\
\text { CIÓN EN } \\
\text { VOLUNTARI } \\
\text { ADO (PV) }\end{array}$ & $\begin{array}{l}\text { PARTICIPACI } \\
\text { ÓN EN } \\
\text { GRUPOS DE } \\
\text { INTERÉS } \\
\text { (PU) }\end{array}$ & $\begin{array}{c}\text { PARTICIPACIÓN EN } \\
\text { ACCIONES } \\
\text { COLECTIVAS } \\
\text { DESAFIANTES (ACD) }\end{array}$ & $\begin{array}{l}\text { PARTICIPACIÓN } \\
\text { EN ASOCIACIONES } \\
\text { RELIGIOSAS (AR) }\end{array}$ \\
\hline Sexo & $-.16 * * *$ & $-.12 * *$ & n.s & $.09 *$ \\
\hline Nivel estudios & $.08^{*}$ & n.s. & $.14 * * *$ & n.s. \\
\hline Nivel ingreso & $.15 * * *$ & $0.1^{*}$ & $.13 * * *$ & n.s. \\
\hline $\begin{array}{l}\text { Infraestructuras } \\
\text { asociativas }\end{array}$ & $.24 * * *$ & $.26 * * *$ & $.22 * *$ & $.16 * * *$ \\
\hline Inseguridad & n.s. & n.s. & n.s. & n.s. \\
\hline $\begin{array}{l}\text { Bienestar } \\
\text { personal }\end{array}$ & n.s. & n.s. & n.s. & n.s. \\
\hline
\end{tabular}

15 Disponibles en: <http://www.capital-social.com/wp/? page_id=251\&preview=true>. Acceso en: 22 jun. 2011. 
A SITUAÇÃO DAS AMÉRICAS:

DEMOCRACIA, CAPITAL SOCIAL E EMPODERAMENTO

\begin{tabular}{|c|c|c|c|c|}
\hline $\begin{array}{l}\text { Solidaridad e } \\
\text { identificación }\end{array}$ & $.60 * * *$ & $.59 * * *$ & $.17 * * *$ & n.s. \\
\hline $\begin{array}{l}\text { Sentido } \\
\text { comunitario }\end{array}$ & $.21 * * *$ & $.24 * * *$ & n.s. & .08 \\
\hline $\begin{array}{l}\text { Apoyo a la } \\
\text { democracia }\end{array}$ & $.09 *$ & n.s. & $.14 * * *$ & n.s. \\
\hline $\begin{array}{l}\text { Participación en } \\
\text { voluntariado }\end{array}$ & --- & $.87 * * *$ & $.24 * * *$ & $.14 * * *$ \\
\hline $\begin{array}{l}\text { Participación en } \\
\text { grupos de } \\
\text { interés }\end{array}$ & $.87 * * *$ & --- & $.21 * * *$ & $.15^{* * *}$ \\
\hline $\begin{array}{l}\text { Participación en } \\
\text { acciones } \\
\text { colectivas } \\
\text { desafiantes }\end{array}$ & $.24 * * *$ & $.21 * * *$ & --- & $.13^{* * *}$ \\
\hline $\begin{array}{l}\text { Participación en } \\
\text { asociaciones } \\
\text { religiosas }\end{array}$ & $.14 * * *$ & $.15 * * *$ & $.13 * * *$ & n.s. \\
\hline $\begin{array}{l}\text { Confianza en } \\
\text { elites }\end{array}$ & $.19 * * *$ & $.16 * * *$ & n.s. & $.12 * * *$ \\
\hline $\begin{array}{l}\text { Confianza } \\
\text { organizaciones } \\
\text { informales }\end{array}$ & $.37 * * *$ & $.29 * * *$ & $.62 * * *$ & $.2 * * *$ \\
\hline $\begin{array}{l}\text { Confianza } \\
\text { organizaciones } \\
\text { formales }\end{array}$ & $.11^{*}$ & $.08^{*}$ & $.09 *$ & $.08^{*}$ \\
\hline $\begin{array}{l}\text { Confianza en } \\
\text { grandes } \\
\text { organizaciones }\end{array}$ & $.17 * * *$ & $.14 * *$ & $.22 * * *$ & $.01 *$ \\
\hline $\begin{array}{l}\text { Confianza en los } \\
\text { medios de } \\
\text { comunicación }\end{array}$ & n.s. & n.s. & n.s. & n.s. \\
\hline $\begin{array}{l}\text { Confianza en } \\
\text { otra gente }\end{array}$ & $.26 * * *$ & $.24 * * *$ & $.13 * *$ & $.09 * *$ \\
\hline $\begin{array}{l}\text { Confianza en } \\
\text { instituciones }\end{array}$ & $.13 * * *$ & $.13 * * *$ & n.s. & $.13^{* * *}$ \\
\hline $\begin{array}{l}\text { Participación } \\
\text { política }\end{array}$ & $.15^{* *}$ & $.12 * *$ & $.22 * *$ & $.1^{*}$ \\
\hline $\begin{array}{l}\text { Discutir sobre } \\
\text { política }\end{array}$ & $.2 * * *$ & $.19 * * *$ & $.31 * * *$ & $.12 * * *$ \\
\hline Informarse & $.14 * * *$ & $.14 * * *$ & $.13 * * *$ & n.s. \\
\hline
\end{tabular}

Nivel de significación: n.s. indica $>0,1 ; *$ indica $\mathrm{p}<0,1 ; * *$ indica $\mathrm{p}<0,05 ; * * *$ indica $\mathrm{p}<0,01$

Fuente: Encuestas de elaboración propia y bases de datos de la pesquisa: Social Capital and Local Participation in South America: Data, Facts and Análisis ${ }^{16}$.

${ }_{16}$ Disponibles en: <http://www.capital-social.com/wp/?page_id=251\&preview=true>. Acceso en: 22 jun. 2011. 
La participación convencional (política, electoral; debatir e informarse) se correlacionaba positivamente con el asociativismo. Entre participación comunitaria (asociaciones de voluntariado y utilitarias) y las acciones colectivas desafiantes había un alto grado relativo de correlación, mayor que en algunas sociedades postindustriales (ver datos en WELZEL et al., 2005). Por otra parte, las mismas correlaciones justificaban ubicar a Chile entre las sociedades en desarrollo y las postindustriales (correlación entre 0,21 y 0,23). Las correlaciones positivas observadas entre voluntariado y diversas formas de confianza reafirman el supuesto de la literatura que a mayor confianza, mayor es la disposición a participar (BAQUERO, 2005).

Algunas variables actitudinales (cultura política) parecen haber tenido relación con la participación. De un modo muy destacado, la solidaridad e identificación social con bienes colectivos tendía a correlacionarse positivamente con la participación comunitaria.

Cuadro 7 - Correlaciones de confianza, variables socioeconómicas y de cultura política (correlaciones, 2005)

\begin{tabular}{|c|c|c|c|c|c|c|}
\hline & $\begin{array}{l}\text { Confianza } \\
\text { en otra } \\
\text { gente } \\
\text { (ICOG) }\end{array}$ & $\begin{array}{l}\text { Confianza } \\
\text { en elites } \\
\text { (ICE) }\end{array}$ & $\begin{array}{l}\text { Confianza en } \\
\text { organizaciones } \\
\text { informales } \\
\text { (ICOI) }\end{array}$ & $\begin{array}{l}\text { Confianza en } \\
\text { orgnizacio- } \\
\text { nes formales } \\
\text { (ICOF) }\end{array}$ & $\begin{array}{c}\text { Confianza } \\
\text { en } \\
\text { grandes } \\
\text { organiza- } \\
\text { ciones } \\
\text { (ICGO) }\end{array}$ & $\begin{array}{c}\text { Confian- } \\
\text { za en } \\
\text { institu- } \\
\text { ciones } \\
\text { (ICI) }\end{array}$ \\
\hline Sexo & $-.081 *$ & n.s. & n.s. & n.s. & $-.075^{*}$ & n.s. \\
\hline Nivel estudios & n.s. & n.s. & n.s. & n.s. & n.s. & n.s. \\
\hline Nivel ingreso & $.21 * *$ & n.s. & $.17 * * *$ & n.s. & $.18^{* * *}$ & n.s. \\
\hline $\begin{array}{l}\text { Infraestructuras } \\
\text { asociativas }\end{array}$ & $.21 * * *$ & n.s. & $.26 * * *$ & n.s. & n.s. & n.s. \\
\hline Inseguridad & $-.22 * * *$ & $-.13 * *$ & $-.09 *$ & $-.16 * * *$ & $-.09 *$ & $-.27 * * *$ \\
\hline $\begin{array}{l}\text { Bienestar } \\
\text { personal }\end{array}$ & $.23 * * *$ & $.18^{* * *}$ & $.1^{*}$ & $.21 * * *$ & $.09 *$ & $.35 * * *$ \\
\hline Calidad de vida & $.16 * * *$ & $.09 *$ & $.11^{*}$ & $.14 * *$ & $.14 * *$ & $.21 * * *$ \\
\hline $\begin{array}{l}\text { Expectativas de } \\
\text { vida }\end{array}$ & $.15^{* *}$ & $.11^{*}$ & n.s. & $.16 * * *$ & $.15^{* *}$ & $.27 * * *$ \\
\hline $\begin{array}{l}\text { Satisfacción con } \\
\text { la democracia }\end{array}$ & $.23 * * *$ & $.28 * * *$ & $.18 * * *$ & $.33 * * *$ & $.27 * * *$ & $.77 * * *$ \\
\hline $\begin{array}{l}\text { Solidaridad } \\
\text { identificación }\end{array}$ & $.72 * * *$ & $.12 * *$ & $.12 * *$ & $.15^{* *}$ & n.s. & $.14 * *$ \\
\hline $\begin{array}{l}\text { Sentido } \\
\text { comunitario }\end{array}$ & --- & $.18^{* * *}$ & $.39 * * *$ & $.33 * * *$ & $.19 * * *$ & $.27 * * *$ \\
\hline $\begin{array}{l}\text { Apoyo a la } \\
\text { democracia }\end{array}$ & $.08 *$ & n.s. & $.14 * *$ & n.s. & n.s. & n.s. \\
\hline $\begin{array}{l}\text { Confianza en } \\
\text { otra gente }\end{array}$ & $.18^{* * *}$ & --- & $.19 * * *$ & $.21 * * *$ & $.15^{* *}$ & $.39 * * *$ \\
\hline $\begin{array}{l}\text { Confianza en } \\
\text { elites }\end{array}$ & $.39 * * *$ & $.19 * * *$ & --- & $.25 * * *$ & $.26 * * *$ & $.25^{* * *}$ \\
\hline Confianza & $.33 * * *$ & $.21 * * *$ & $.25 * * *$ & --- & $.27 * * *$ & $.61 * * *$ \\
\hline
\end{tabular}


A SITUAÇÃO DAS AMÉRICAS:

DEMOCRACIA, CAPITAL SOCIAL E EMPODERAMENTO

\begin{tabular}{|c|c|c|c|c|c|c|}
\hline $\begin{array}{l}\text { organizaciones } \\
\text { informales }\end{array}$ & & & & & & \\
\hline $\begin{array}{l}\text { Confianza } \\
\text { organizaciones } \\
\text { formales }\end{array}$ & $.19 * * *$ & $.15^{* *}$ & $.26 * * *$ & $.27 * * *$ & --- & $.34 * * *$ \\
\hline $\begin{array}{l}\text { Confianza en } \\
\text { grandes } \\
\text { organizaciones }\end{array}$ & $.27 * * *$ & $.39 * * *$ & $.25 * * *$ & $.61^{* * *}$ & $.34 * * *$ & --- \\
\hline $\begin{array}{l}\text { Confianza en } \\
\text { instituciones }\end{array}$ & $.12 * *$ & $.18^{* * *}$ & $.14^{* *}$ & $.23 * * *$ & $.15^{* *}$ & $.36 * * *$ \\
\hline $\begin{array}{l}\text { Confianza en los } \\
\text { medios de } \\
\text { comunicación }\end{array}$ & $.17 * * *$ & $.27 * * *$ & $.29 * * *$ & $.17^{* * * *}$ & $.51 * * *$ & $.21^{* * *}$ \\
\hline $\begin{array}{l}\text { Participacion } \\
\text { politica }\end{array}$ & $.14 * *$ & $.09 *$ & $.27 * * *$ & $.08^{*}$ & $.34 * * *$ & n.s. \\
\hline $\begin{array}{l}\text { Discutir sobre } \\
\text { política }\end{array}$ & $.09 *$ & n.s. & $.15^{* *}$ & n.s. & $.23 * * *$ & n.s. \\
\hline Informarse & n.s. & n.s. & n.s. & n.s. & n.s. & n.s. \\
\hline
\end{tabular}

Fuente: Encuestas de elaboración propia y bases de datos de la pesquisa: Social Capital and Local Participation in South America: Data, Facts and Análisis ${ }^{17}$.

Sumariamente, las variables de participación, vida asociativa y la confianza tienen correlaciones nítidas entre sí, superiores al promedio, en todos los casos. A su turno, las confianzas tienen correlaciones considerables con la vida asociativa y entre sí. Asimismo, se observa algunas correlaciones de interés entre la vida asociativa, la confianza y otras variables socioeconómicas, de calidad de vida y de cultura política. La correlación entre el asociativismo y la actitud de solidaridad e identificación social es particularmente alta, es decir, un porcentaje significativo de las personas que participan manifiestan actitudes de identificación y de solidaridad con sus bienes colectivos. De la misma forma, desde la perspectiva de la confianza, se observa una alta correlación entre la confianza en otra gente y el sentido de la responsabilidad social colectiva. Aquí podría haber un recurso social importante para la participación ciudadana y el desarrollo local.

\section{Condiciones para una participación ciudadana efectiva y habilitantes: propuestas para la discusión}

Las apreciaciones con respecto a las políticas públicas chilenas de descentralización, fortalecimiento de los gobiernos locales y participación ciudadana, y sus debilidades, sumadas a las evidencias sobre los bajos niveles de vida asociativa, confianza y dimensiones más libres y espontaneas de la

17 Disponibles en: <http://www.capital-social.com/wp/? page_id=251\&preview=true>. Acceso en: 22 jun. 2011. 
participación ciudadana (informarse, debatir) sugieren un gran problema de fondo: Chile aún carece de una buena sintonía entre la oferta estatal de espacios e instrumentos para la participación y los intereses, inquietudes y demandas por vida asociativa y participación ciudadana.

Si las instituciones, las políticas y los programas dejan de cumplir las funciones para las cuales han sido concebidas, entonces se justifica la reforma. En el caso de Chile, en lugar de seguir imperando los mismos supuestos conceptuales, métodos e instrumentos que han dado lugar a políticas de descentralización y participación ciudadana, corresponde innovar, cambiar y mejorar. Es más, de persistir enfoques y políticas públicas que no consideran las realidades donde la vida asociativa ocurre y la participación ciudadana genuina es posible, probablemente haya que esperar una crisis, tal como ha sucedido en otros países de la región, para buscar una reingeniería estructural en una situación de emergencia extraordinaria.

A la luz de los magros niveles de vida asociativa y confianza observados en Chile, cabe preguntar si el país dispone realmente de una institucionalidad adecuada en tamaño, estructura, competencias, instrumentos, y los mecanismos apropiados y pertinentes para acoger el asociativismo y la participación ciudadana, en cuanto condiciones enriquecedoras de la democracia y el desarrollo sustentable. En la dirección de innovar y mejorar, la última sección de este estudio consiste en propuestas que apuntan a ir perfeccionando una serie de condiciones necesarias para que las políticas de participación tengan la posibilidad de ser efectivas y habilitantes en los espacios locales.

\section{Coordinación intersectorial y profesionalismo}

Las instituciones del Estado deben mejorar la coordinación y calidad de sus intervenciones para obtener mejores resultados en términos de participación y confianza. Las prácticas de múltiples intervenciones sectoriales, costosas y dispersas, deben ser sustituidas por una metodología rigurosa que priorice la coordinación, el trabajo profesional y las economías de escala. Las instituciones del estado deben intervenir en aquellos ámbitos donde tengan competencias técnicas y profesionales, sin tratar de abarcarlo todo, dejando un espacio abierto para que las propias comunidades que son objeto de sus intervenciones asuman un papel protagónico, al igual que otros actores de la sociedad civil.

\section{Perspectiva de genuina promoción de la participación}

La acción de los ministerios, agencias y servicios del Estado central en las regiones, muy especialmente en los municipios, no debiese limitarse a la instalación de programas y servicios pensados y diseñados bajo una lógica centralista. No obstante ser agentes externos que no habitan los territorios 
donde ejecutan programas, sus intervenciones, cuales quiera que sean, debiesen ir a la par de un proceso dirigido a instalar y reforzar recursos y capacidades, transferir herramientas e información, de tal modo que los actores locales asuman un rol activo de agentes en la toma de decisiones, con conocimiento, responsabilidad y resguardo de los intereses colectivos.

Todos los programas del Estado en las comunas del país debiesen tener explícitamente la intencionalidad de involucrar a la ciudadanía local y sus organizaciones en el diseño de las políticas locales, en una suerte de gestión "integrada" que convoque e involucre en la esfera de lo público, más allá del Estado en cuanto sistema central de toma de decisiones.

Metodológicamente, la aproximación que se produce entre el Estado y la comunidad debe superar el modelo causal "identificación de problemaspropuesta de solución". La nueva aproximación metodológica debe partir por reconocer el potencial existente en las localidades, sus particularidades, con el propósito de poner en práctica estrategias que favorezcan la participación y el desarrollo sustentable. Cambiar la forma de aproximación de entrada implica repensar, rediseñar instrumentos y hábitos de acercamiento, terminar con prácticas de interacción contaminadas por enormes asimetrías de información y con criterios discrecionales y utilitaristas al servicio de logros cortoplacistas, y por sobre todo generar instancias reales de diálogo y trabajo conjunto. Este cambio tan simple en el modo de hacer las cosas incentivará el desenvolvimiento de la vida asociativa, la confianza y la participación en espacios de autonomía y autogestión de las personas y sus organizaciones.

La generación de confianzas entre los actores y su reconocimiento es fundamental para la sustentabilidad de los resultados. De no invertirse en la inclusión y participación de ellos desde un comienzo, no serán viables los acuerdos y los compromisos colectivos en el futuro. Este proceso no debiese ser considerado como una pérdida de tiempo y de recursos frente a la presión que impone el medio ambiente y los tiempos y ritmos de las autoridades de turno y la necesidad de mostrar resultados al corto plazo. Dada la importancia de la confianza y la cooperación para la participación, los actores estatales, centrales y municipales, deben conocer bien los territorios donde intervienen, identificar a los actores claves en él; tanto dirigentes, personas reconocidas y valoradas por la comunidad, organizaciones formales y funcionales, instituciones con permanencia y presencia en la comuna. Esta actividad ayudará a generar redes de apoyo y de cooperación que den sustentabilidad a los proyectos.

Una comunidad bien informada, donde existen espacios de participación definidos y conocidos por todos, demandará cada vez más espacios y canales que le permita ser parte de la toma de decisiones sobre las políticas que le afectan. Habrá por tanto una tendencia creciente a involucrarse más y más en los asuntos públicos, en la medida que la invitación a participar sea transparente, abierta y razonable. Más aún, la claridad e información sobre las reglas y el procedimiento facilitan el 
involucramiento con responsabilidad, incentivan la motivación y generan expectativas acordes a los logros esperados.

La generación de confianza va de la mano con la transparencia en las comunicaciones y en los procedimientos. Especialmente en relación con la gestión en las municipalidades y en las organizaciones comunitarias, donde están en juego el uso de recursos públicos y decisiones que tienen consecuencias para todos, son indispensables los mecanismos que faciliten la información de la comunidad, no solo en relación con los propósitos y grandes metas de los programas e instancias gubernamentales de participación, sino también con los beneficios y procedimientos para acceder a ellos, cómo pueden incorporarse las personas en distintas fases, y con qué consecuencias concretas.

\section{Transferencia de capacidades y competencias}

Los procesos de participación y desarrollo resultarán favorecidos mediante el proceso formativo de fortalecimiento de las competencias en las personas. El proceso puede incluir acciones de educación formal y de capacitación, tales como son educación a distancias, talleres, encuentros, pasantías, giras, ferias, entre otros. En esa línea, las personas debiesen tener acceso a tecnologías que les permitan ser independientes en la búsqueda de nuevos conocimientos e informaciones de utilidad.

\section{Superación de asimetrías de información}

La información es uno de los factores más relevantes para la participación en los procesos de toma de decisión, porque confiere fundamentos, competencias cognitivas, procedimentales y habilitantes para la intervención. Contar con información de calidad no sólo enriquece las propuestas, los diseños y la ejecución de los programas, sino también otorga coherencia a los procesos de toma de decisión de las autoridades y de las comunidades, reforzando la legitimidad de las acciones del Estado.

La generación y distribución de información de calidad para la participación y la confianza es una actividad costosa para los entes públicos. Una alternativa consiste en perfeccionar los mecanismos y acciones integradas para generar y procesar información, por una parte, y progresar gracias al uso de herramientas tecnológicas que den la posibilidad de integrar información dispersa, y faciliten diagnósticos y análisis al servicio de las intervenciones en los espacios comunales, por otra. En las realidades comunales chilenas, caracterizadas por la heterogeneidad, la acción integrada que incentive la asociación de las voluntades de las instituciones públicas, las comunidades y otros actores locales, en torno a metas y acciones concretas, generará sinergia para una buena gobernabilidad. Por su parte, las tecnologías de la información están en condiciones de ofrecer plataformas 
tecnológicas propicias para un trabajo más coordinado y profesional que en el pasado.

La acción integrada y las plataformas tecnológicas debiesen facilitar los sistemas de monitoreo para el seguimiento de un conjunto acotado de observaciones de acceso público que faciliten las intervenciones de los actores que deseen colaborar en las políticas de participación, cooperando de manera integrada un sistema regular de observación de la participación. Por medio de esta práctica, se irá generando un lenguaje común de la participación en los distintos niveles que influyen en ámbito local y las sinergias necesarias.

\section{Estándares de participación}

El progreso en la dirección de políticas integradas de participación que entren en sintonía con inquietudes ciudadanas, vida asociativa y confianza, requiere un consenso básico sobre los principales parámetros de participación, del cual deben participar diversos actores en diferentes niveles del Estado y de la sociedad. Esos parámetros deben ser operacionalizados en forma de instrumentos de observación. Finalmente, la observación y la información de la participación debiesen estar al servicio del seguimiento autónomo de todos los involucrados.

¿Qué observar? Como punto de partida proponemos incluir un conjunto de prácticas vinculadas a determinadas dimensiones que permitan saber cómo está funcionando realmente el sistema de la participación ciudadana local. En este sentido es necesario observar un conjunto acotado hechos que den cuenta de una multiplicidad de procesos de participación social que ocurren en el nivel local. A partir de ellos, se elaborarán indicadores que entregarán información objetiva sobre el estado de la participación en ese lugar. La observación de prácticas participativas consistiría en observar las conductas y las acciones de funcionarios, ciudadanos y de todos quienes intervienen en el ámbito local. Asimismo, la observación debiese incluir procedimientos y condiciones, esto es, factores influyen en las acciones de participación en calidad de variables intervinientes, ya sea como infraestructura u otros factores que influyen en la accesibilidad a la información.

\section{Conclusión}

Los antecedentes expuestos dejan en evidencia que el Estado de Chile dispone efectivamente de instrumentos de participación ciudadana y de incentivo de la vida asociativa que podrían ser perfeccionados, en la dirección de entrar en una mayor sintonía con las inquietudes ciudadanas. En necesario examinar críticamente y reformar aquello que funciona mal, y por sobre todo abrir espacios para que la participación ciudadana, la vida asociativa y la confianza se desplieguen en libertad y con autonomía. 
Como bien hemos señalado en la actualidad el desafío está puesto en lograr un "gobierno cercano a la gente" que conjugue en forma virtuosa el contacto permanente con la población y los niveles crecientes de efectividad en la puesta en marcha de instrumentos que respondan a las reales necesidades de los ciudadanos y sus comunidades. En ese contexto, el municipio, siendo la cara más visible del aparato estatal frente a la ciudadanía, se ve enfrentado a la necesidad de satisfacer demandas concretas y urgentes de las personas y, por otra parte, desarrollar procesos inclusivos en la búsqueda de soluciones, con un énfasis en la calidad de resultados que sintonicen con inquietudes ciudadanas.

Para enfrentar en forma eficiente su función, el gobierno local deberá avanzar hacia la identificación las demandas de manera integral y especializada territorialmente, enfrentando con esta nueva lógica la tradicional forma de intervenir sectorialmente, desconociendo las particularidades y potencialidades de un territorio y su población.

Además, a esa tarea se suma la de reinventar y validar nuevos espacios que vayan en la línea de la habilitación y la participación cogestionaria de proyectos colectivos. Ello requiere fortalecer las competencias de intervención de los ciudadanos y de la comunidad con herramientas favorezcan la participación responsable, que incluye compromisos de largo plazo, y también por medio políticas que legitimen su accionar a través de prácticas transparentes y abiertas a todos. Para ello, habrá que luchar contra la presión por mostrar estados de avances visibles y tangibles que impide desarrollar un soporte robusto, a partir del cual se desplieguen procesos de diseño y ejecución que sumen a la ciudadanía a un proyecto común y desafiante.

Patricio Valdivieso é Licenciado em História pela Pontificia Universidad Catolica de Chile e Doutor em História e Ciência Politica pela Katholische Universitaett Eichataett, na Alemanha. Atualmente é Pesquisador e Docente na Universidade de Los Lagos e Universidade Bernardo O'Higgins, Santiago Chile.

E-mail: pvaldivf@gmail.com

\section{Referencias}

BAEZA F., Rodrigo. Manual Jurídico Municipal. Santiago: CPU, 2004.

BAQUERO, Marcello. Um modelo integrado de democracia social na América Latina. Revista Debates, Porto Alegre, v. 1, n. 1, (número extraordinário), p. 5-55, 2005. 
CARMONA, Erika. Participación de la comunidad en los asuntos públicos de Chile: Una mirada desde el espacio local (1990-2005). Diálogo Político, Buenos Aires, s/v., n. 1, p. 1-28, mar. 2006.

COLEMAN, James S. Foundations of Social Theory. Cambridge: Cambridge University Press, 1990.

NORRIS, Pippa. Democratic Phoenix: Reinventing Political Activism. Cambridge: Cambridge University Press, 2002.

PUTNAM, Robert D. Making democracy work: Civic traditions in modern Italy. Princeton: Princeton University Press, 1993.

SUBDERE. Subsecretaría de Desarrollo Regional. Participación ciudadana en la gestión de gobiernos regionales y municipios: diagnóstico actual. Santiago: SUBDERE, 2004.

VALDIVIESO, Patrício. Propuesta para el progreso de la administración pública y de la gestión local con participación ciudadana, desde la perspectiva del caso de Chile. Documentos y Aportes en Administración Pública y Gestión Estatal, Santa Fe, s/v., n. 11, p. 89-123, jul.-dez. 2008.

. Capital Social e Desenvolvimento Democrático: Porto Alegre (Brasil) e Santiago do Chile. Revista Brasileira de Ciências Sociais, São Paulo, v. 24, n. 69, p. 93-114, fev. 2009.

VALDIVIESO, Patricio et. al. Justicia y sociedad civil: el papel de la sociedad civil en la reforma judicial: estudios de casos en Argentina, Chile, Colombia y Perú. Buenos Aires: INECIP, 2003.

WELZEL, Chistian et. al. Social Capital, Associational Life, and Collective Action: What Makes Some Publics More 'Civic' than Others? In: APSA - The American Political Science Association, Annual Meeting. Washington D.C., 2005. 